This report was prepared as an account of work sponsored by an agency of the United States Government. Neither the United States Government nor any agency thereof, nor any of their employees, makes any warranty, express or implied, or assumes any legal liability or responsibility for the accuracy, completeness, or usefulness of any information, apparatus, product, or process disclosed, or represents that its use would not infringe privately owned rights. Reference herein to any specific commercial product, process, or service by trade name, trademark, manufacturer, or otherwise does not necessarily constitute or imply its endorsement, recommenoation, or favoring by the United States Goyernment or any agency thereof. The views and opinions of authors expreased herein do not necessarily state or reflect those of the United States Government or any agency thereor.

N. K. Glendenning

Nuclear Science Division Lawrence Berkeley Laboratory

University of California

Berkeley, California 94720

This work was supported by the Director,

Office of Energy Research,

Division of Nuclear Physics of the Office of High Energy and

Nuclear Physics of the U.S. Department of Energy

under Contract No. DE-AC03-76SF00698. 


\title{
SOLITON MATTER AS A MODEL OF DENSE NUCLEAR MATTER
}

\author{
N. K. Glendenning \\ Nuclear Science Division \\ Lawrence Berkeley Laboratory \\ University of California \\ Berkeley, California 94720
}

Abstract

We employ the hybrid soliton model of the nucleon consisting of a topological meson field and deeply bound quarks to investigate the behavior of the quarks in soliton matter as a function of density. To organize the calculation, we place the solitons on a spatial lattice. The model suggests the transition of matter from a color insulator to a color conductor above a critical density of a few times normal nuclear density. 
SOLITON MATTER AS A MODEL OF DENSE NUCLEAR MATTER

\author{
N. K. Glendenning \\ Nuclear Science Division \\ Lawrence Berkeley Laboratory \\ University of California \\ Berkeley, California 94720
}

A great deal of interest has focussed recently on solitons as representing non-perturbative solutions of $Q C D$ for baryons [1]. A number of authors have shown that at the $30 \%$ level, solitons resemble nucleons $[2]$. What we find particularly appealing in this development is that, having a Lagrangian that describes the internal structure of the nucleon (soliton), one can investigate interesting questions concerning how the internal structure changes when solitons are assembled to form dense matter, and how the properties of matter correspondingly change. Several of the more interesting questions concern the quark behavior in normal and in dense matter, such as the anomalous muon scattering on nuclei as compared to nucleons (EMC effect) $[3]$, and the onset of deconfinement. Of course we will not believe literally the predictions of the theory. It is in the large $\mathrm{N}_{\mathrm{c}}$-limit that QCD becomes equivalent to an effective field theory of mesons, and counting rules suggest that baryons may emerge as solitons $|1|$. We live in a three color world and I know of no criteria by which we may judge how far from the limit we are, aside from the empirical success at the $30 \%$ level that has been claimed by a number of authors for soliton models of the nucleon. In any case I do not expect that our model of matter as consisting of solitons will rival the lattice gauge calculations for quantitative predictions of phase transitions. However, when a physical theory is very complex, as is the theory of strong interactions, it is always useful to have a model with which to form at least qualitative pictures of how the theory works. The model may suggest ways of probing nature that the exact theory, solved on large computers may not do. In this paper I will report on the start that several of us have made on such a program [4]. It is far from finished and there remain serious problems to be overcome so this is a progress report. 
But before introducing our model of matter, I will recall for you some of the salient features of soliton models of the nucleon. First of all, what is a soliton? Briefly, it is a solution to a non-linear theory whose energy density has a finite spatial extent, and which is stable in the sense that if several soliton solutions are constructed in different regions of space and allowed to come into proximity so that they interact, after they have moved apart they are restored to their original form.

It was Skyrme who first suggested that baryons might be understood as soliton solutions of a field theory having only mesons as the fields [5] . How do baryons emerge from a theory that has only meson fields? I can't make that altogether convincing, but it is easy to see that an anomalous integer quantum number emerges, which Skyrme conjectured to be baryon number, and which Witten recently confirmed [1]. Skyrme studied a theory based on a scalar and triplet of pi mesons, constructing the two by two matrix,

$$
\mathrm{U}=\frac{1}{\mathrm{f}_{\mathbf{r}}}\left(\begin{array}{cc}
\sigma+\pi_{3} & \mathrm{i} \pi_{1}+\pi_{2} \\
-\mathrm{i} \pi_{1}+\pi_{2} & \sigma-\pi_{3}
\end{array}\right)=\sigma(\mathrm{x})+\underset{\sim}{\mathrm{i} \tau} \cdot \underset{\sim}{\pi(\mathrm{x})}
$$

and from this the quantity,

$$
\mathbf{L}_{\mu}=\mathrm{U}^{+} \partial_{\mu} \mathrm{U}
$$

Skyrme constructed the Lagrangian,

$$
\mathscr{L}=\frac{\mathrm{f}_{\mathrm{r}}^{2}}{4} \operatorname{tr}\left[\mathrm{L}_{\mu} \mathrm{L}^{\mu}\right]+\frac{\eta^{2}}{4} \operatorname{tr}\left[\mathrm{L}_{\mu}, \mathrm{L}_{\nu}\right]^{2} .
$$

The first term is an unfamiliar way of writing the more familiar meson part of the non-linear sigma model $[6]$. It turns out that there are no stable finite size soliton solutions for a theory possessing only the first term, and the second term was added to provide stability against collapse of the solution. I want to draw attention to the fact that it is of fourth order in derivatives of the fields, and that a sixth order term or higher would also stabilize the solution. This term plays no other essential role. In particular the quantum number is unaffected by it. The reason 
for drawing attention to this arbitrary form will emerge at the end of the paper.

To show that there is a soiiton solution, one makes the very peculiar ansatz that a solution of the form,

$$
\mathrm{U}_{0}=\mathrm{e}^{\mathrm{i} \tau \cdot \hat{\mathrm{i}} \theta(\mathrm{r})}=\cos \theta(\mathrm{r})+\underline{\tau} \cdot \underline{\hat{\mathrm{r}}} \sin \theta(\mathrm{r})
$$

exists. That is, that the isospin components of the pion field point in the spatial radial direction,

$$
\sigma=\mathrm{f}_{\pi} \cos \theta(\mathrm{r}) \quad, \quad \underline{\pi}=\underline{\underline{\mathrm{I}}} \mathrm{f}_{\boldsymbol{\pi}} \sin \theta(\mathrm{r})
$$

For that reason the solution is called the hedgehog. It is not my purpose to proceed to show that this is a solution. That can be done easily by calculating the canonical form of the energy from the Lagrangian, substituting the ansatz for the fields, and minimizing. This yields an equation for the chiral angle, $\theta(r)$, which has a solution that smoothly connects the boundary values

$$
\theta(0)=-\mathbf{n} \pi \quad, \quad \theta(\infty)=0 .
$$

With these bounary values, the energy is finite and can be seen to be localized in the vicinity of the origin where $\theta(r)$ is non-vanishing.

What I do want to draw attention to is that in addition to the Noether currents that correspond to the invariances of the Lagrangian, the theory possesses an anomalous current,

$$
B_{\mu}=\frac{1}{24 \pi^{2}} \varepsilon_{\mu \alpha \beta \gamma} \operatorname{tr}\left[L^{\alpha} L^{\beta} L^{\gamma}\right]
$$

where $\varepsilon_{\mu \alpha \beta \gamma}$ is the antisymmetric tensor in all indices. By construction this quantity is divergenceless,

$$
\partial_{\mu} \mathbf{B}^{\mu}=0
$$

and the charge, corresponding to the ansatz (5) and the boundary conditions (6) is 


$$
\begin{aligned}
B & =\int d^{3} r B_{0}(r)=\frac{1}{\pi}\left[\theta(r)-\frac{1}{2} \sin 2 \theta(r)\right]_{0}^{\infty} \\
& =n
\end{aligned}
$$

This soliton solution has therefore a conserved quantity which is integer, and is associated with the non-trivial mapping of isospin space and 3-space represented by (4). Therefor B is called the topological charge, and it is what Skyrme conjectured to be baryon number.

My purpose in reviewing this material was to introduce the conserved topological charge, which you see is associated with the SU(2) character of the theory, and will carry cver to other modifications of the theory. The Skyrmion as such is not interesting to us for the purpose set out in the beginning, because it has no quarks, and we want to see how the quarks begin to leak out of the baryons as the density of matter is increased. This is perhaps relevant both to the deconfinement phase transition as well as to anomalous lepton scattering from nuclei (EMC effect). Therefor, we would like to have a soliton with quarks that are confined, but not through the artificial mechanism of an impervious bag. In the absence of a known soliton solution possessing true confinement, we opt for a model in which the quarks are deeply bound in a topological soliton field. The hybrid soliton model fills this requirement $[7,8]$. The hybrid soliton, like the Skyrmion, is based on the chiral sigma model, but now including the fermion sector, which here are quarks. In the limit of large scalar meson mass, the Lagrangian is,

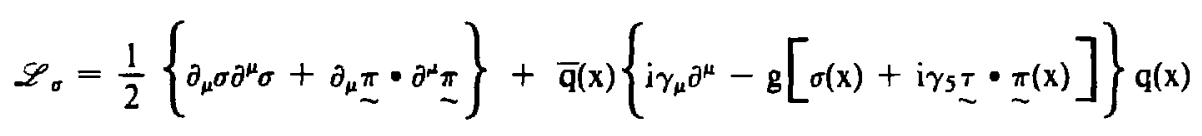

This consists of the first term of (3) and in addition the Lagrangian of the quarks, with spinors $q(x)$, which are Yukawa coupled to the scalar and pion fields. What Kahana et al [7], and Birse and Banerjee [8] showed is that there is a solution in which the quarks are deeply bound to the topological soliton field of the mesons, and for which the mass of the object is the nucleon mass. They were uncertain about the baryon charge of the object however. The soliton carries 
its conserved topological charge, which has been identified with baryon number, while the three quarks carry a baryon charge. The authors of ref. [7] concluded that the hybrid soliton has $\mathrm{B}=2$, for this reason. However, we shall show below how to identify the charge as $B=1$. First however I need to tell you how the field equations that follow from (10) can be solved, in the mean field approximation. For the hedgehog configuration (5) there is one non-linear differential equation for the chiral angle $\theta(r)$ which is coupled to the Dirac equation for the quark spinors. The latter is,

$$
\left\{i \gamma_{\mu} \partial^{\mu}-m\left(\cos \theta(r)+i \gamma_{5} \underline{\sim} \cdot \underline{\underline{r}} \sin \theta(r)\right)\right\} q(x)=0
$$

where $m=g f_{\pi}$ is the constituent quark mass. The Dirac equation has a solution of the form,

$$
q(\underline{r})=\left.\left(\begin{array}{c}
F(r) \\
i \sigma \cdot \underline{\sim} \underline{\underline{r}} G(r)
\end{array}\right)\right|_{\nu>}
$$

where $\nu>$ is a spinor eigenstate of the sum of spin and isospin, having eigenvalue zero,

$$
(\underline{\sigma}+\underset{\sim}{\tau}) \mid \nu>=0
$$

That such a peculiar coupling of spin and isospin occurs, follows from the coupling of the quarks to the hedgehog meson field in which the isospin components of the pion field point in the radial direction. The differential equations for the $F$ and $G$ are,

$$
\begin{aligned}
-\mathrm{F}^{\prime}+\mathrm{m} F \sin \theta(\mathrm{r}) & =(\mathrm{E}+\mathrm{m} \cos \theta(\mathrm{r})) \mathrm{G} \\
\mathrm{G}^{\prime}+\left(\frac{2}{\mathrm{r}}+\mathrm{m} \sin \theta(\mathrm{r})\right) \mathrm{G} & =(\mathrm{E}-\mathrm{m} \cos \theta(\mathrm{r})) \mathrm{F}
\end{aligned}
$$

We call the state (12) a positive parity state after the transformation of the large component. The state of opposite parity satisfies equations like (14) but with $\mathrm{m} \rightarrow-\mathrm{m}$. The eigenstates (12) are triply (color) degenerate.

I shall not write the equation for $\theta(\mathrm{r})$. Kahana et al solved the coupled equations numerically, with the boundary conditions (6), and they also found that a good representation for $\theta(\mathrm{r})$ 
is given by,

$$
\theta(r)= \begin{cases}\pi\left(r / R_{s}-1\right) & , \quad r<R_{s} \\ 0 & r \geqslant R_{s}\end{cases}
$$

where we can call $R_{s}$ the soliton size. It is found by minimizing the total energy of the soliton (quarks + meson fields). Of particular interest to us at this point is the behavior of the triply degenerate quark eigenvalues as a function of $R_{s}$. This is indicated schematically in Fig. 1. At this point we can discuss the baryon charge on the soliton. For large $R_{s}$, the $0+$ state falls to the vicinity of $-\mathrm{m}$. That is to say, the spectrum of Dirac states appears as the usual free states with a gap between $\mathrm{m}$ and $-\mathrm{m}$. We shall consider the configuration in which the negative energy sea is completely occupied. The filled negative sea has by definition no baryon charge. The meson field however carries one unit of topological charge. Now as we shrink the soliton the $0+$ state rises, even to positive energy for small enough $\mathbf{R}$. In any case we add no additional quarks to the system. The same states throughout are occupied. Therefor the baryon number remains unchanged, and equal to one, the value of the topological charge.

Now we wish to assemble a large number of such solitons to form dense matter. You can appreciate on reflection that this poses on intractable problem as it stands. It is a many-body problem in which the quarks within the individual solitons are moving in interaction with each other through the meson fields, while the solitons are moving about under the influence of the interaction of their constituent quarks with those of neighbouring soliton fields. We shali therefor study a particular possible ground state configuration of matter, one in which the solitons are arranged on a lattice. The short-range repulsion between the solitons, (refer to Fig. 1 to see how the valence quark level rises as the soliton is squeezed), makes this a plausible configuration. Since the valence quarks are deeply bound, by of the order of their constituent mass, they are relativistic. We have therefor a relativistic solid state problem.

As an initial orientation on what to expect, we solved for the Dirac equation, a problem that had been solved long ago for the Schroedinger equation by Kronig and Penny, namely the 
eigenvalue spectrum for fermions in a periodic square well potential in one-dimension. For the potential shown in Fig. 2, the analytic solution is given by,

$$
\frac{\mathrm{Q}^{2}-\mathrm{K}^{2}+\mathrm{V}^{2}}{2 \mathrm{QK}} \sinh 2 \mathrm{Qb} \sin 2 \mathrm{Ka}+\cosh 2 \mathrm{Qb} \cos 2 \mathrm{Ka}=\cos 2 \mathrm{k}(\mathrm{a}+\mathrm{b})
$$

where,

$$
Q^{2}=(m+V)^{2}-\varepsilon^{2} \quad, \quad K^{2}=\varepsilon^{2}-m^{2}
$$

and $\mathrm{k}$ is the so-called crystal momentum. In the limit $\varepsilon \rightarrow \mathrm{m},(16)$ goes over to the nonrelativistic formula of Kronig and Penny. The allowed values of the particle energy are those for which the left side does not exceed in absolute value, unity, so that the spectrum has the well known band structure. A typical spectrum as a function of the spacing between the attractive regions is shown in Fig. 2. The parameters of the problem are chosen so that the fermions become relativistic toward the top of the well. As in the non-relativistic case, the levels of the isolated wells, spread out into bands with each well contributing a level to each band. For close spacing, the bands tend to touch. The band structure persists into the positive energy spectrum above the top of the potential, with the gaps tending toward zero as the energy increases. Kelativity complicated the problem, but it did not introduce any qualitative changes.

We turn now to the solution of the problem at hand, the spectrum of quarks in soliton crystal matter. The hedgehog meson configurations are centered at lattice points thus generating a periodic field in which the quarks move. From solid state physics we know that the solution of the Hamiltonian for a periodic system must obey Bloch's theorem. Therefor the quark spinor must be of the form,

$$
\underline{q_{k}}(\underline{r})=e^{i k-r} \cdot \underline{u_{k}}(r)
$$

where $\mathbf{k}$ is called the crystal momentum and $u_{k}(r)$ is a periodic spinor function having the period of the lattice. That is to say, the solutions are modulated plane waves. 
To solve (14) on the lattice we employ the Wigner-Seitz approximation. Thus the actual problem is replaced by a spherically symmetric one which is solved for $k=0$, which is the ground state of the band. For convenience we shall make an ansatz for the behavior of the chiral angle, similar in spirit to Kahana et al. [7]. Denote by $R_{s}$ the equilibrium radius of the isolated soliton. When the lattice spacing, $2 \mathrm{R}$, between solitons exceeds the diameter of a soliton,

$$
\theta(r)=\left\{\begin{array}{lll}
\pi\left(r / R_{s}-1\right) & , & r<R_{s} \\
0 & R_{s} \leqslant r \leqslant R
\end{array}, \quad R>R_{s}\right.
$$

Otherwise,

$$
\theta(\mathbf{r})=\pi(\mathbf{r} / \mathbf{R}-1) \quad, \quad \mathbf{R} \leqslant \mathbf{R}_{\mathbf{s}}
$$

For the ground state of the band, the periodicity of the Schroedinger wave function required by the Bloch theorem imposes the condition that it have zero slope at the Wigner-Seitz boundary. This requirement translates in the case of the Dirac equation to

$$
F^{\prime}(R)=0 \quad, \quad G(R)=0
$$

i.e. ine large componert has zero slope and the small component, zero value at the boundary, as follows from (14) and (19). At the origin, it is evident from (14) that $G(0)=0$. This in turn requires that $F(0)=0$. Therefor the boundary conditions

$$
\mathrm{G}(0)=\mathrm{G}(\mathrm{R})=0
$$

ensure that the Bloch theurem is satisfied, i.e. that both $F$ and $G$ are periodic. We solve the coupled Dirac equations (14) with the boundary condition in the Wigner-Seitz cell (21), by numerical integration. The eigenvalues for the $0+,-$ states are shown in fig 3 . The lower of these two belongs to the filled sea of quarks, and the other is the valence orbital. This orbital as would be expected from the Schrodinger theory, is lowered in energy from the isolated soliton eigenvalue over a certain range of crystal spacings, and then it rises due to the compression of the solitons by their neighbours. The lower eigenvalue is increased for all crystal spacings, 
which behavior can be traced to the small component of the Dirac spinor when the eigenvalue is close to $-\mathrm{m}$.

The Wigner-Seitz approximation allows us to calculate the eigenvalue of the ground state of each band $(k=0)$, Denote such an eigenvalue for a particular band by $e_{0}$. We need to estimate the band width. In the Schroedinger theory this is done by calculating follows the expectation value of the energy of the state, $k$, which is $e_{0}+k^{2} / 2$. One could do the same in the Dirac case, and find $\varepsilon_{\mathrm{k}}=\sqrt{\varepsilon_{0}^{2}+\mathrm{k}^{2}}$ with $\mathrm{k}=0$ to $\pi / 2 \mathrm{R}$. Alternately we are motivated by the tight binding approximation of solid state physics. We calculate the eigenvalue for isolated solitons, ie with exponentially decaying boundary condition, but with chiral field given by (21). The band width is then estimated as twice the difference between this energy and that computed with the crystal boundary condition, because the band should be spread symmetrically about the unperturbed case. For the valence levels the Wigner-Seitz approximation locates the bottom of the band. However for the levels belonging to the sea, it locates the top of the band, just as the sea eigenvalues in the free case are $-\sqrt{\mathbf{m}^{2}+\mathbf{k}^{2}}$.

The band structure is shown in fig 3 by the shaded areas and the solid lines are the Wigner-Seitz eigenvalues $(k=0)$. Several points deserve comment. We see a lowering of the valence quark eigenvalue by about $16 \mathrm{MeV}$ at a lattice spacing $2 \mathrm{R}=2.45 \mathrm{fm}$. For smaller spacing the level rises steeply and the top of the band intersects the continuum at a spacing of about $1 \mathrm{fm}$, which corresponds to a density of 7 times normal nuclear density $\left(.15 / \mathrm{fm}^{3}\right)$. Since the occupied triply degenerate levels of the isolated solitons are filled, so too the occupied bands of the crystal are filled, and it is an insulator. However at that density where the top of an occupied band passes into the continuum, matter ceases to be an insulator and becomes increasingly a color and electric conductor as the density is further increased.

The above behavior is suggestive of quark deconfinement, although in this model the quarks are not truly confined but only deeply bound in the isolated state. The wave functions of the sea and valence orbitals are shown for a typical lattice spacing in fig. 4 , illustrating their 
periodicity in the crystal. In fig. 5, we compare the quark distribution in soliton matter of several densities, illustrating the increasing concentration at the cell boundary for increasing density. For the pion decay constant we employ the experimental value $f_{\pi}=93 \mathrm{MeV}$, and a coupling constant $\mathrm{g}=7.55$, which yields a soliton mass of $966 \mathrm{MeV}$, close enough to the nucleon mass for our purpose.

During the course of this work another paper has been published which investigated Skyrmion matter in a crystal lattice approximation [9]. This model however does not possess quarks. Nevertheless, as these authors point out, the asymptotic behavior of the equation of state is such that the energy density behaves like $\rho^{4 / 3}$, just as a relativistic gas of Fermions. This is also the behavior in the model studied here, since the quarks pass into the continuum states of dense matter. We point out however, that for the Skyrmion, this behavior is an artifact of the form chosen to stabilize the Skyrmion, namely a term of fourth order in derivatives and hence in $\mathrm{k} \sim \boldsymbol{\rho}^{1 / 3}$. This is the lowest order stabilizing term, and can be viewed as the first in a series, the last of which will dominate the momen 'um dependence of the equation of state at high density.

In summary, we have investigated the behavior of quarks in soliton matter, using the hybrid model consisting of a topological meson field and deeply bound quarks. To organize the calculation, we placed the solitons in a crystal lattice. This trial configuration is justified at high density because of the repulsion of the topological solitons. At a certain critical density, the top of the valence quark band becomes degenerate with the Fermi sea, meaning that the quarks in those states are no longer bound to a lattice site. At still higher densities; additional levels of the band rise into the continuum, suggesting that color conductivity is a gradual function of compression. 
Acknowledgements

This work was supported by the Director, Office of Energy Research, Division of Nuclear Physics of the Office of High Energy and Nuclear Physics of the Department of Energy under Contract DE-AC03-76SF00098. 
References

1. G. t’Hooft, Nucl. Phys. B72 (1974) 461; B75 (1974) 461.

E. Witten, Nucl. Phys. B160 (1979) 57; B223 '1983) 433.

2. M. Rho, A. S. Goldhaber and G. E. Brown, Phys Rev. Lett. 51 (1983) 747.

G. S. Adkins, C. R. Nappi and E. Witten, Nucl. Phys. B228 (1983) 552.

A. Jackson, A.D. Jackson and V. Pasquier, (Stonybrook Preprint, 1984).

R. Vinh Mau, M. Lacombe, B. Loiseau, W. Cottingham and P. Lisboa, (Orsay Preprint, 1984).

3. J. J. Aubert et. al., Phys. Lett. 123B (1983) 275.

4. B. Banerjee, N. K. Glendenning and V. Soni LBL-18644.

5. T. H. R. Skyrme, Proc. Roy. Soc. A260 (1961) 127.

6. M. Gell-Mann and M. Levy, Nuovo. Cim. 6 (1960) 705.

7. S. K. Kahana, G. Ripka and V. Soni, Nucl. Phys. A415 (1984) 351.

8. M. C. Birse and M. K. Banerjee, Phys. Lett. 136B (1984) 284.

9. M. Kutschera, C. J. Pethick and D. G. Ravenhall, Phys. Rev. Lett. f3 (1984) 1041. 
Figure 1. Schematic of quark eigenvalues in topological meson field as a function of soliton radius. (adapted from [7])
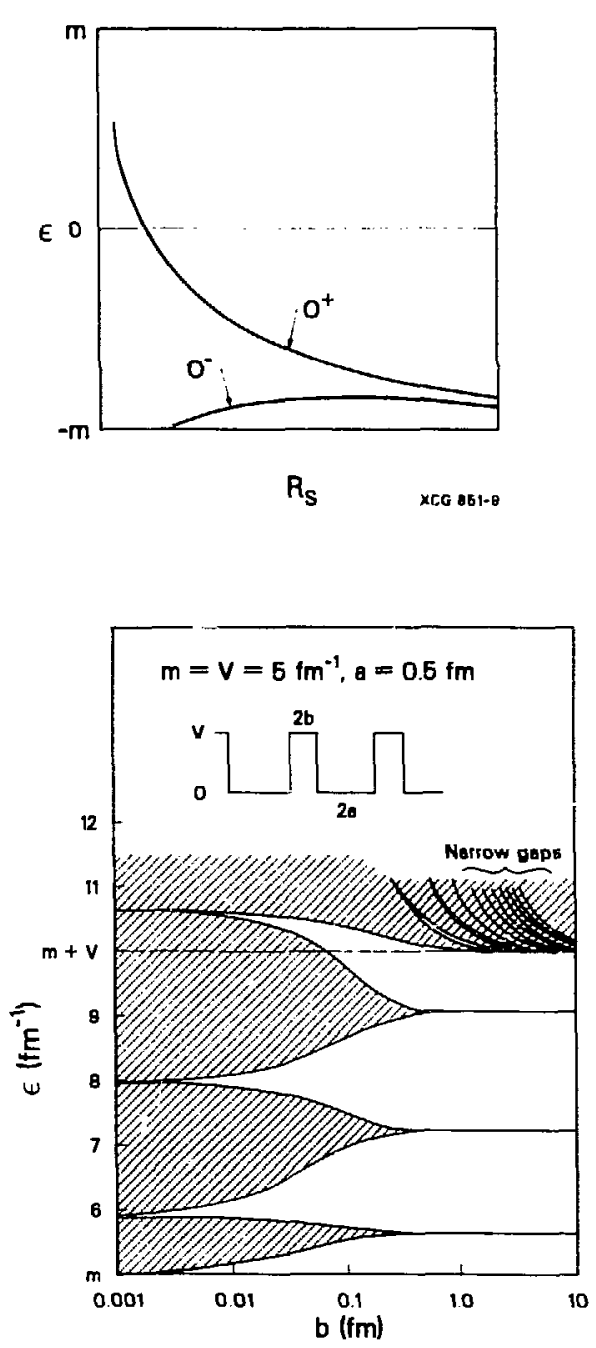

XCG $851-10$

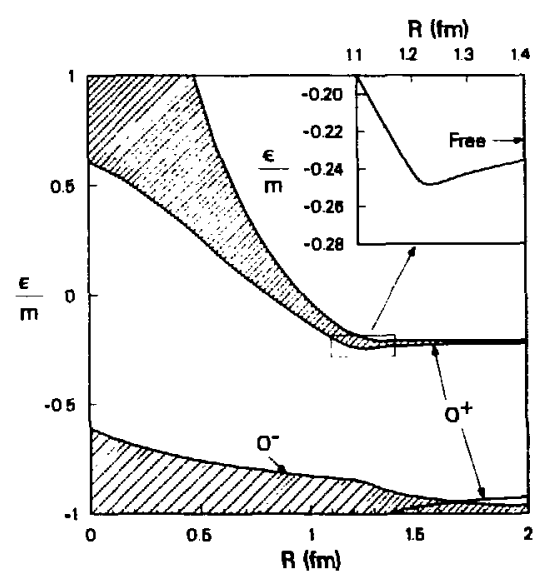

Figure 2. Energy level band structure of relativistic particles in a one-dimensional square well lattice as a function of spacing between the attractive regions. This is a graphical representation of the analytic solution.

Figure 3. Eigenvalues of the valance $(0+)$ and sea (0-) orbitals of quarks in soliton matter as a function of Wigner-Seitz cell radius, $R$. The band of levels that develops as the spacing decreases is shown by the shaded region. In the upper right corner, an enlargement of the region indicated is shown. The eigenvalue of a free soliton is indicated by the arrow. 
Figure 4. Upper (F) and lower (G) components of the Dirac spinor are shown for the valence and sea orbitals for a cell radius of $0.6 \mathrm{fm}$.

Figure 5. Probability distribution for the valence quarks for several cell radii, illustrating the increasing concentration of quarks at the cell boundary as the compression of soliton matter increases.
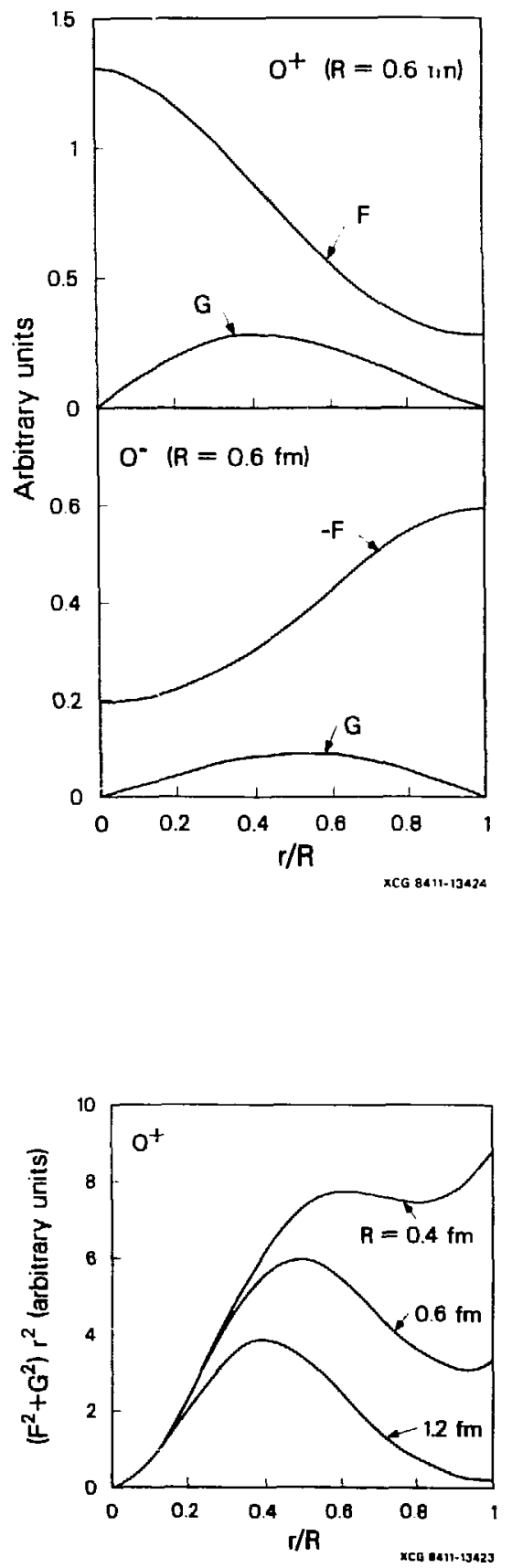
This report was done with suppor from the Department of Energe. Any conclusiors or opinions expressed in this report repiesent solely those of the author(s) and not necessarily those of The Regents of the Liniversity of California, the Lawrenc= Berkeley Laboratory or the Depariment of Energy:

Reference 10 a company or produst name does nat imply approval or recommendation of the product by the University of Califomia or the U.S. Department of Ensrgy to the exclusion of others that may be suitable. 